\title{
Using Laser Microfabrication to Write Conductive Polymer/SWNTs Nanocomposites
}

\author{
Qingchuan GUO $^{{ }^{*}}$, Shizhou XIAO ${ }^{* 1}$, Andreas AUMANN ${ }^{* 1}$, Matthias JAEGER ${ }^{* 1}$, M'Barek CHAKIF ${ }^{* 1}$, Reza GHADIRI ${ }^{{ }^{1}}$, \\ Cemal ESEN ${ }^{* 1}$, Mingyan MA ${ }^{* 2}$, Andreas OSTENDORF ${ }^{* 1}$ \\ ${ }^{* 1}$ Ruhr-University Bochum, Laser Applications Technology, Universitaetsstr. 150, 44801 Bochum, \\ Germany \\ Email address: guo@lat.rub.de,Andreas.Ostendorf@ruhr-uni-bochum.de \\ ${ }^{* 2}$ Ruhr-University Bochum, Inorganic Chemistry I, Universitaetsstr. 150, 44801, Bochum Germany
}

\begin{abstract}
We present a novel laser microfabrication method to generate structures on the basis of a new class of functional photosensitive composites. In particular, the focus lies on the development of conductive composites by incorporating SWNTs into the matrix of polymers thus forming highly conductive nanocomposites. Conductive microstructures have been produced with the ultra-high resolution technology based on laser direct writing (e.g. single-, two-photon polymerization) using polymer/SWNTs nanocomposites. This technology opens new prospects for the realization of novel MEMS and MOEMS with increased functionality, integration, and higher level of miniaturization.
\end{abstract}

DOI:10.2961/jlmn.2012.01.0008

Keywords: Laser micro- and nanofabrication, Polymer matrix, Polymer/Single-Walled Carbon Nanotubes (SWNTs) Nanocomposites, Structures, Conductivity

\section{Introduction}

Laser micro- and nanofabrication technology [1,2] has been proven as one of the promising approaches to the manufacturing of microoptical components, micronanomachines, and their integrated systems $[3,4]$. However, because most 3-D microstructures made of photosensitive polymers are non-conductive by nature, their use in MEMS and MOEMS e.g. as electrostatic actuators is limited. To overcome this limitation, we present our investigations into the fabrication of conductive microstructures by means of laser direct writing on polymer/SWNTs (Single-Walled Carbon Nanotubes) nanocomposites. SWNTs have been proven to successfully be implemented into the matrix of polymers to form conductive nanocomposites. The effective utilization of SWNTs in composite applications strongly depends on the ability to disperse them homogeneously throughout the matrix [5-7]. In this paper we present a number of laser microfabrication methods to fabricate high quality conductive structures. For this we pursue two steps. The first step is to develop a homogeneous dispersion of nanocomposites by direct mixture of polymer and SWNTs with different SWNTs concentrations, subsequently optimize the suitable nanocomposite concentration, i.e. realization of polymerization process at specific laser wavelength. The second step involves the generation of microstructures using laser micro- and nanofabrication. We generated structures using the single-photon polymerization (1PP, stereolithography) process induced by picosecond laser pulses and two-photon polymerization (2PP) effect induced by NIR femtosecond laser pulses.

\section{Development of homogeneous nanocomposites}

The development of homogeneous nanocomposites is critical in the field of polymer/SWNTs composites and significant efforts have been made to enhance the dispersibility of SWNTs in a polymer matrix [8]. In this paper we make use of the high conductivity of SWNTs (Nanoamor Inc. $\sigma>10^{4} \mathrm{~S} / \mathrm{m}$ ) which are directly doped into the low conductivity polymer matrix (OrmocersOrmocore ${ }^{\circledR}$ b59 with $1.8 \%$ Photoinitiator Irgacure from Microresist technology $\mathrm{GmbH} \sigma \approx 10^{-14} \mathrm{~S} / \mathrm{m}$ ) to tailor the electrical and structural properties suitable for different applications.

\subsection{Samples preparation}

Polymer/SWNTs composites with a concentration range between 0.005 and 5.0 wt.-\% were prepared, by direct mixing pure SWNTs into the liquid polymer (exsitu preparation). The mixtures were first stirred for $24 \mathrm{~h}$ at $70^{\circ} \mathrm{C}$ by magnetic stirring and then sonicated by a bath-type sonicator for different duration at $50^{\circ} \mathrm{C}$. Polymer/SWNTs composites were sonicated for $60 \mathrm{~min}$, $75 \mathrm{~min}, 90 \mathrm{~min}, 105 \mathrm{~min}$, and $300 \mathrm{~min}$. Subsequently, the mixtures were sonicated again for $15 \mathrm{~min}$ at room temperature. As the dispersion efficiency of the SWNTs is strongly affected by the viscosity of the polymer the sonication of the SWNTs carried out at higher temperature was found to provide a homogeneous dispersion, while sonication at room temperature could not produce homogeneous composites, instead, the SWNTs were aggregated [9].

After the homogeneous composites have been achieved, the samples will be prepared in the following steps: a small drop of the composite was placed between two PET films where the distance between two films was controlled by a spacer film $(200 \mu \mathrm{m})$ placed between 
them. UV light from a high-pressure mercury lamp irradiated the composite for various times $(1-10 \mathrm{~min})$ depending on the concentration of the SWNTs. The composite films (Figure 1) were characterized after peeling them off from the PET films. Raman spectroscopy measurements were recorded using Renishaw Raman Microscope, and four-point probe was used to measure the conductivity.

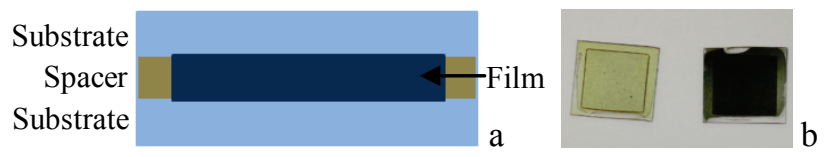

Fig. 1: Schematic for the composite film preparation (a) and photograph of composite films at $0.01 \mathrm{wt.} \%$ (left) and $3.0 \mathrm{wt.}-\%$ (right) concentration with size $12 \mathrm{~mm} \times 12 \mathrm{~mm} \times 200 \mu \mathrm{m}$ (b).
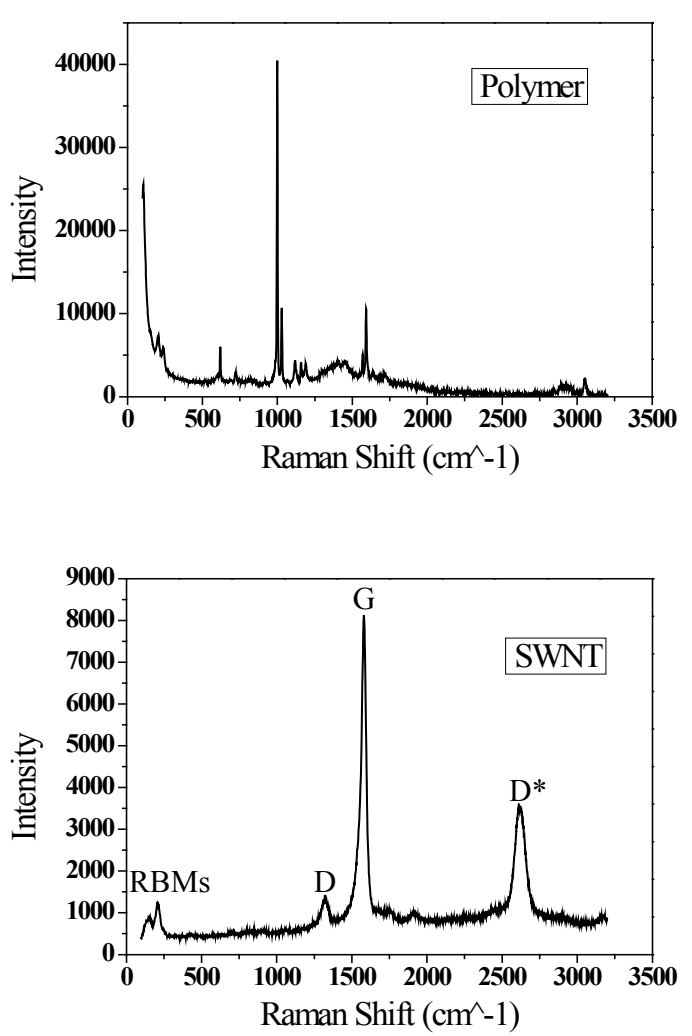

Fig. 2: Raman spectra of pristine polymer (top) and raw SWNTs (bottom): The characteristic peaks correspond to diameter dependent radial breathing mode (RBM) and disorderinduced D, D* and tangential G band of SWNTs.

\subsection{Raman spectroscopy measurements}

The Raman spectra of the films at an excitation wavelength of $785 \mathrm{~nm}$ are shown in the following figures (2-4). By use of Raman spectroscopy measurements in order to examine the influence of the polymer matrix on the unique properties of the SWNTs, especially the electrical properties of the SWNTs after mixing and polymerization might be changed, Raman spectra of the two components were recorded in advance and after processing. As shown in figure 2, the Raman spectra of pristine polymer and raw SWNTs that were prepared to compare with the results after processing. Figure 3 and 4 show the Raman spectra of the processed samples. The spectra are almost identical to the superposition of pristine polymer and raw SWNTs. The upper right figures of figure 3 and 4 for the G-Band area provide that all samples exhibit almost the same peaks. The results show that grafting of the polymer onto the sidewall of the SWNTs did not occur and the SWNTs remain intact during the polymerization in the present system.

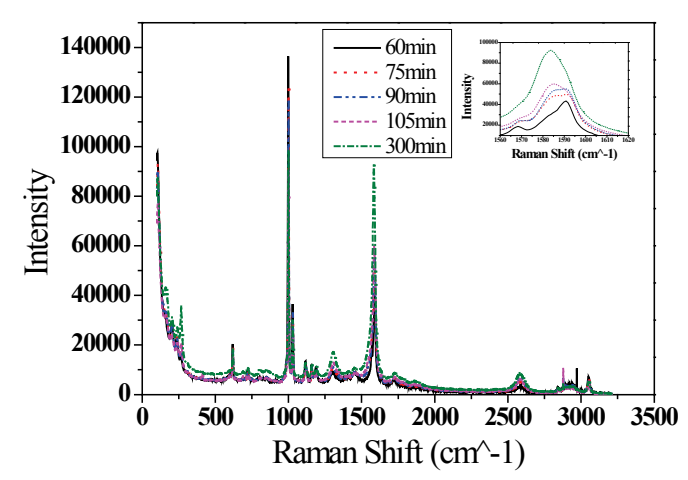

Fig. 3: Raman spectra of SWNTs (1.0 wt.-\%)-composite at different sonication time. The upper right figure shows in GBand area all samples at different sonication time have almost the same peaks.

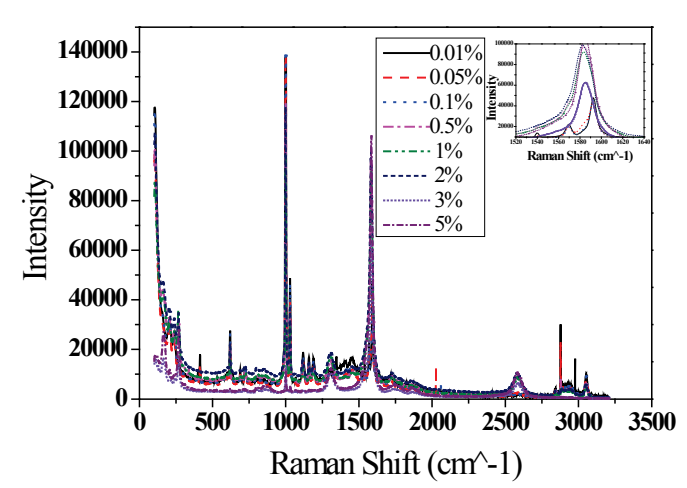

Fig. 4: Raman spectra of SWNTs/polymer composites at different concentration. The upper right figure shows in G-Band area all samples with different concentration have almost the same peaks.

\subsection{Conductivity measurements}

The presence of the conductive SWNTs within an insulating matrix material can alter the electrical properties of composites $[9,10]$. The composite becomes conductive above a critical value percolation threshold that defines the insulator-conductor transition. The electrical percolation threshold depends on many factors including the size and shape of the SWNTs, matrix properties, preparation method, SWNTs properties, and dispersion of the SWNTs within matrix. The dispersion is shown in figure 5. 

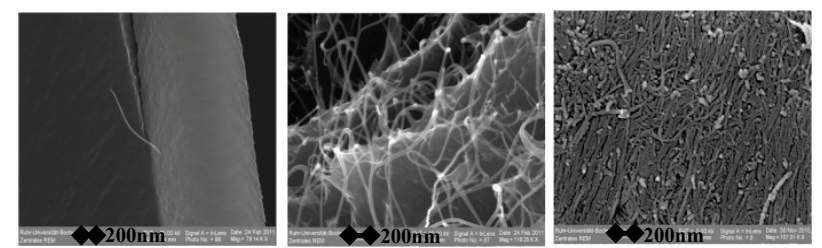

Fig.5: Dispersion of composites: bad dispersion, without SWNTs no conductivity (left); Electrical percolation threshold (center) is observed when SWNTs are sufficiently close to each other to form a percolating conductive path; SWNTs are aggregated (right), the laser beam could not transmit through the aggregated SWNTs.

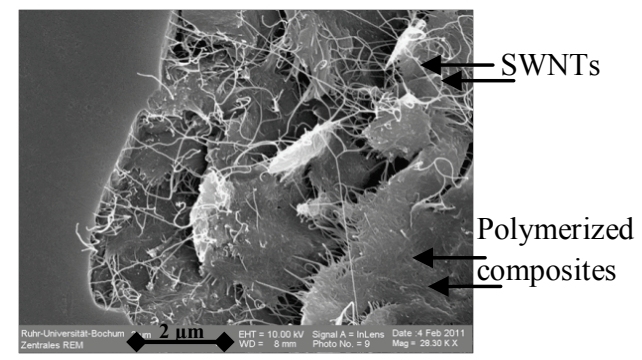

Fig. 7: Cross-section of polymerized composite after breaking, most of SWNTs are localized inside of film.

Table 1: Conductivity unless at 2.0 wt.-\% concentration and long sonication time. After polished with a removal layer of about $10 \mu \mathrm{m} \mathrm{105*}$ min, the conductivity increased. * Beyond measuring range of device.

\begin{tabular}{|c|c|c|c|}
\hline Sonication time & 2.0 wt. $-\%$ & 3.0 wt. $-\%$ & 5.0 wt. $-\%$ \\
\hline $60 \mathrm{~min}$ & $*$ & $*$ & $2.35 \mathrm{E}-07 \mathrm{~S} / \mathrm{m}$ \\
\hline $75 \mathrm{~min}$ & $*$ & $*$ & $8.96 \mathrm{E}-07 \mathrm{~S} / \mathrm{m}$ \\
\hline $90 \mathrm{~min}$ & $*$ & $*$ & $4.15 \mathrm{E}-07 \mathrm{~S} / \mathrm{m}$ \\
\hline $105 \mathrm{~min}$ & $*$ & $*$ & $1.29 \mathrm{E}-07 \mathrm{~S} / \mathrm{m}$ \\
\hline $105^{*} \min$ & $3.81 \mathrm{E}-08 \mathrm{~S} / \mathrm{m}$ & $2,53 \mathrm{E}-07 \mathrm{~S} / \mathrm{m}$ & $9.70 \mathrm{E}-07 \mathrm{~S} / \mathrm{m}$ \\
\hline $300 \mathrm{~min}$ & $3.57 \mathrm{E}-08 \mathrm{~S} / \mathrm{m}$ & $9.05 \mathrm{E}-07 \mathrm{~S} / \mathrm{m}$ & $3.03 \mathrm{E}-07 \mathrm{~S} / \mathrm{m}$ \\
\hline
\end{tabular}

Electrical conductivity can be used as one of the useful properties of polymer/SWNTs composites for evaluating the degree of the SWNTs dispersion and developing possible applications of the composites [11], as the effective use of polymer/SWNTs composites depends on the homogeneous dispersion [5-7]. In our research a four-point probe was used to measure the conductivity of all samples after polymerization. At room temperature the conductivities of composite films that contain different concentrations and sonication times were measured and are summarized in table 1. The composites only at high concentration and long dispersion time exhibit sufficient conductivity. However, the conductivity was found improved from 2.0 wt.- $\%$ after the film of the composite surface was polished with a removal layer of about $10 \mu \mathrm{m}$ (Figure 6), as shown for the samples at sonication time of $105^{*} \mathrm{~min}$. This indicates that the surface of polymerized composites is pure polymer, most of SWNTs are dispersed inside the film (Figure 7). In summary, our work revealed that the nanocomposites have conductivity unless at $2.0 \mathrm{wt} .-\%$.
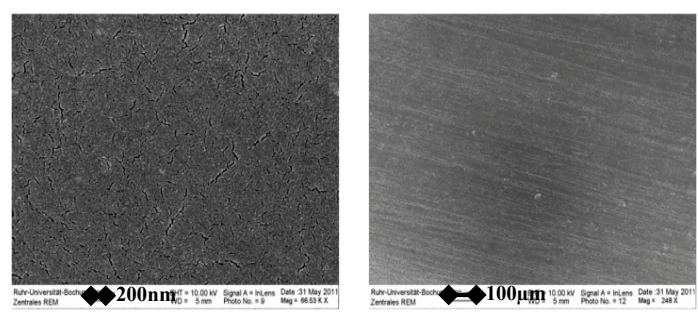

Fig. 6: Surface before (left) and after (right) removing a layer of about $10 \mu \mathrm{m}$.

\section{Results using laser micro- and nanofabrication}

The structures were fabricated using laser micro- and nanofabrication by illuminating a film of a liquid composite on the cover glass. The laser beam is moved across the sample by using a scanner and focused by a F-Theta objective. After illumination, the unpolymerized composite has been washed away with a developer OrmoDev (Microresist technology $\mathrm{GmbH}$ ). The structures were positioned by using an online observation system with a camera. In this paper both $1 \mathrm{PP}$ and $2 \mathrm{PP}$ structures have been written. The difference between 1PP and $2 \mathrm{PP}$ is that $1 \mathrm{PP}$ with the absorption of the laser at the surface of the UV photosensitive polymer and 2PP with the focusing and intensity-driven absorption inside the polymer. Due to this fact, $1 \mathrm{PP}$ is a 2-dimensional process which can generate 2.5-dimensional structures by packing layer by layer. The advantage of $2 \mathrm{PP}$ process, compared to $1 \mathrm{PP}$, is the possibility for the generation of arbitrary 3-dimensional micro- and nanostructures.

\subsection{Single-Photon Polymerization (1PP) Results}

2-dimensional structures were fabricated using the $1 \mathrm{PP}$ process. The samples were written by means of a picosecond UV laser (LUMERA LASER GmbH; wavelength $\lambda=355 \mathrm{~nm}$, repetition rate $\mathrm{f}=200 \mathrm{KHz}$ ). The laser fluence was $70.8 \mathrm{~mJ} / \mathrm{cm}^{2}$, writing speed was set to $10 \mathrm{~mm} / \mathrm{s}$.

Composites within the investigated concentration range could be properly polymerized. Figure 8 shows the structures at low and high concentration of composites. The surface of the structures from composites at low concentration is perfectly smooth. In contrast, composites with higher concentration show a rough surface 
indicating that there are some aggregated SWNTs on the surface of structures. In this case the laser beam could be heavily scattered by aggregated SWNTs which can damp the polymerization process, especially for 3-dimensional processing where transmission at the fundamental laser wavelength is required.
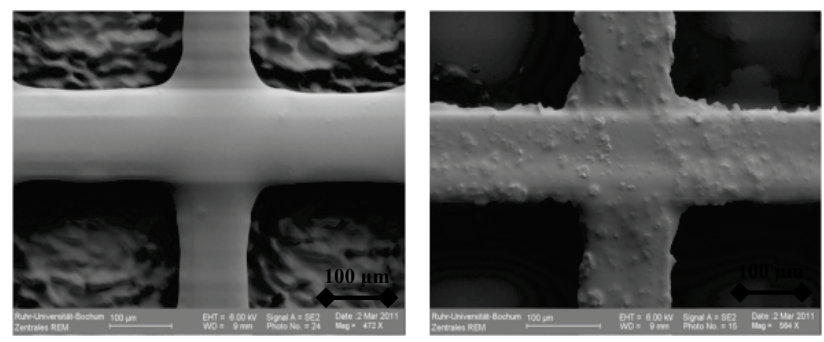

Fig. 8: SEM images of composite structures via $1 \mathrm{PP}$ at 0.01 wt.-\% (left) and 3.0 wt.-\% (right).

\subsection{Two-Photon Polymerization (2PP) Results}

To realize nanostructures, in the present work $2 \mathrm{PP}$ technology was used for photopolymerization of polymer/SWNTs composite. A femtosecond laser system (Spectra Physics Tsunami ${ }^{\circledR}$, wavelength $\lambda=800 \mathrm{~nm}$, delivering pulses of 90 -fs duration at repetition rate $\mathrm{f}=$ $80 \mathrm{MHz}$ ) was used as the light source and a $100 \times$ microscope objective lens (Nikon, Plan Apochromat, N.A. $=1.4$ ) was used to focus the laser beam into the volume of the composite. The laser power was set to $32.6 \mathrm{~mW}$, writing speed was $4 \mathrm{~mm} / \mathrm{s}$.

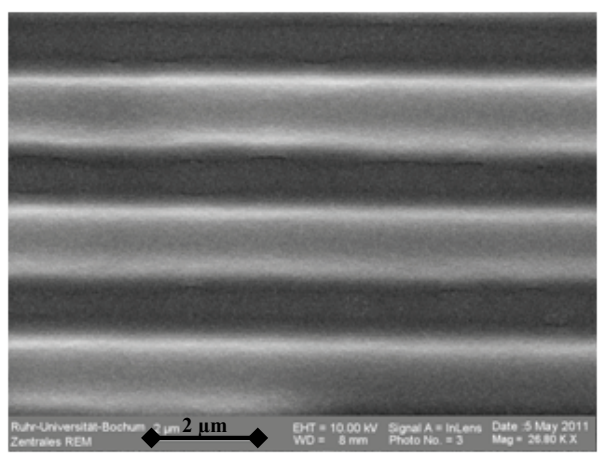

Fig. 9: SEM image of composite structure via $2 \mathrm{PP}$ at 0.01 wt.- $\%$

Figure 9 shows SEM image of polymer/SWNTs composite nanowires fabricated by means of 2PP. After the unpolymerized composite was washed away, solidified structures were left. However, because of the linear absorption of the aggregated SWNTs, photons are feasible to be directly captured by SWNTs instead by photoinitiator molecules. This significantly affects the threshold of 2PP processing [12,13] and parts of the fabricated structures have been shrunken, which have led to the distortion of the fabricated structures, and thus leads to a damping of the $2 \mathrm{PP}$ processing. In order to avoid such distortion, in future work the relevant laser parameters will be optimized and the dispersibility of SWNTs within polymer matrix should be further improved. In addition, the polymerization process should be initiated at the focal point, and the focal point located at the interface between substrate and composites.

\section{Conclusions}

We have presented investigations into functional composites which can be structured by laser direct writing. The conductivity of composite can be principally be improved by increasing the SWNTs concentration and sonication time. Raman spectroscopy measurements have shown that by direct mixture method grafting of the polymer onto the sidewall of SWNTs did not occur, the SWNTs remain intact during the polymerization in the present system. The composites were photopolymerized both at low and high concentration by 1PP processes. At higher concentration the laser beam will be scattered by aggregated SWNTs. It can be concluded that extinction of embedded SWNTs by absorption and scattering will damp the polymerization process, especially for 3dimensional structures based on $2 \mathrm{PP}$ processing. Using 2PP processing we have produced composite structures, however, the structures were still distorted.

In future, first of all, the dispersibility of SWNTs in the polymer matrix will be improved, in order to avoid aggregated SWNTs to damp 2PP processing. Furthermore, we will apply some of the presented methods to investigate and determine the boundaries of SWNTs concentration and 2PP processing. In this way we will gain the suitable SWNTs concentration that the composites can be used as functional and structural polymers.

\section{Acknowledgments}

We would like to thank Deutsche Forschungsgemeinschaft (DFG) for the generous support of this work in the framework of project OS188/17-2.

\section{References}

[1] H. B. Sun and S. Kawata, Adv. Polym. Sci. 170, (2004) 169

[2] S. Maruo, O. Nakamura, and S. Kawata, Opt. Lett. 22, (1997) 132

[3] H. B. Sun, K. Takada, and S. Kawata, Appl. Phys. Lett. 79, (2001) 3173

[4] K. S. Lee, D. Y. Yang, S. H. Park, and R. H. Kim, Polym. Adv. Technol. 17, (2006) 72

[5] M. Meyyappan, "Carbon nanotubes science and applications”; CRC Press: Boca Raton, FL, (2005)

[6] P. J. F. Harris, "Carbon nanotubes and related structures new materials for the twenty-first century'; Cambridge University Press: Cambridge, (2001)

[7] M. J. O'Connell, 'Carbon nanotubes properties and applications"'; CRC Taylor \& Francis: Boca Raton, (2006)

[8] M. Moniruzzaman, K. I. Winey: Macromolecules 39 (16), (2006) 5194-5205

[9] Y. J. Kim, T. S. Shin, H. D. Choi, J. H. Kwon, Y. C. Chung, H. G. Yoon: Carbon 31 (1), (2005) 23-30 
[10] S. Barrau, P. Demont, A. Peigney, C. Laurent, C. Lacabanne: Macromolecules 36 (14), (2003) 51875194

[11] T. Fujigaya, Sh. Haraguchi, T. Fukumaru, and N. Nakashima: Adv. Mater. 20, (2008) 5151-2155
[12]K. Tanaka, H. B. Sun, and S. Kawata, Appl. Phys. Lett. 80, (2002) 312

[13]Z. B. Sun, X. Z. Dong, W. Q. Chen, S. Nakanishi, X. M. Duan, and S. Kawata, Adv. Mater. (Weinheim, Ger.) 20, (2008) 914

(Received: June 17, 2011, Accepted: December 21, 2011) 